\section{Características da violência escolar no Brasil: revisão sistemática de estudos quantitativos}

\section{Characteristics of school violence in Brazil: a systematic review of quantitative studies}

Francine Nesello 1

Flávia Lopes Sant'Anna 2

Hellen Geremias dos Santos 3

Selma Maffei de Andrade 4

Arthur Eumann Mesas 5

Alberto Durán González 6

1-6 Programa de Pós-graduação em Saúde Coletiva. Universidade Estadual de Londrina. Av. Robert Koch, 60.Vila Operária. Londrina, PR, Brasil. E-mail: franesello@gmail.com

\begin{abstract}
Objectives: to measure and identify factors associated with school violence in Brazil, as reported in the literature.

Methods: articles containing quantitative data on school violence in Brazil were selected from the SciELO, LILACS and PubMed databases, up to May 2013. The selection of articles and extraction of data were carried out independently by two researchers and inconsistencies resolved by consensus.

Results: twenty-four studies met the inclusion criteria. Most of these studies were carried out among basic education students in public schools in the South and Southeast regions of Brazil in the 2000s. School violence was defined differently and different types studied in the articles examined. Psychological and physical violence were the most common forms addressed. Bullying was commonly cited as a specific kind of school violence. Being male and having been subjected to abuse in the family were the factors most frequently associated with school violence.

Conclusions: the present review noted the paucity of quantitative studies of violence in Brazilian schools, especially among teachers. There is a need to establish criteria for the study of this phenomenon, in order to compare its geographical and temporal distribution.
\end{abstract}

Key words Bullying, Violence, School health, Review

\section{Resumo}

Objetivos: dimensionar e identificar fatores associados à violência escolar no Brasil descritos na literatura.

Métodos: foram selecionados artigos com dados quantitativos sobre violência escolar no Brasil e indexados nas bases de dados SciELO, LILACS e PubMed, até maio de 2013, sem restrição temporal para o início. A seleção de artigos e a extração dos dados foram realizadas de modo independente por duas pesquisadoras e as inconsistências resolvidas por consenso.

Resultados: vinte e quatro estudos cumpriram os critérios de inclusão. A maioria destes foi desenvolvida com alunos do ensino fundamental em escolas públicas localizadas no Sul e no Sudeste brasileiro e durante os anos 2000. A definição de violência escolar e as modalidades estudadas diferiram entre os artigos analisados. A violência psicológica e a fisica foram as mais abordadas. O bullying destacouse como modalidade específica da violência escolar. Ser do sexo masculino e ter vivenciado situações de violência na familia foram os fatores mais freqüentemente associados à violência escolar.

Conclusões: a presente revisão detectou poucos estudos quantitativos sobre violencia em escolas brasileiras, sobretudo entre professores. É necessário estabelecer critérios para o estudo desse fenômeno, que permitam comparação de sua ocorrência no tempo e no espaço.

Palavras-chave Bullying, Violência, Saúde escolar, Revisão 


\section{Introdução}

A violência é inerente à existência humana e manifesta-se de modo peculiar em espaços sociais distintos. Vista como fenômeno complexo e multifacetado, atinge gama variada de pessoas, grupos, instituições e povos. ${ }^{1}$ Define-se violência como o uso intencional da força física ou do poder, real ou em ameaça, contra si próprio, contra outra pessoa, ou contra um grupo ou uma comunidade, que resulte ou tenha grande possibilidade de resultar em lesão, morte, dano psicológico, perturbação do desenvolvimento ou privação. ${ }^{2}$

O fenômeno da violência contra crianças e adolescentes coloca em destaque o cenário escolar, que é, depois do ambiente familiar, o espaço de maior convívio social desses indivíduos. ${ }^{3}$ StelkoPereira e Williams ${ }^{4}$ ressaltam a delimitação geográfica como um dos elementos chave para a definição de violência escolar. Para essas autoras, essa modalidade de violência é a que ocorre dentro do espaço físico da escola, durante o trajeto casa-escola, em locais onde ocorram passeios e/ou festas escolares programadas e em bairros e residências de alunos cujos assuntos escolares mal resolvidos repercutam em violência.

Nas últimas décadas, tem crescido o interesse em compreender a violência no contexto escolar, não só por suas implicações no processo de integração de crianças e adolescentes à sociedade, mas pela íntima relação que apresenta com o fracasso de objetivos mais amplos da escola, como educar, ensinar e aprender. 5

A violência escolar vem sendo pesquisada em diversos países, tanto no Ocidente6,7 como no Oriente. 7,8 No Brasil, os processos de abertura de escolas e de democratização do acesso à educação, nas últimas décadas, trouxeram implicações importantes à compreensão da violência no contexto escolar. Esse movimento, ao mesmo tempo em que vislumbrou possibilidades de melhores condições de vida para um maior número de indivíduos, colaborou para o aumento da heterogeneidade de comportamentos e de valores, assim como para evidenciar desigualdades no ambiente escolar e para o aumento de conflitos. 9,10

Entre os dados necessários ao conhecimento da violência escolar, medidas de frequência, prevalência e características desse fenômeno são indispensáveis, pois podem colaborar na identificação de ações importantes de serem desenvolvidas no âmbito da saúde pública e da educação, além de fornecer subsídios à adoção de políticas públicas para redução de atos violentos nesses espaços de convivência e aprendizado. Nesse sentido, o objetivo do presente estudo foi caracterizar, dimensionar e identificar fatores associados à violência escolar em estudos realizados no Brasil e publicados em periódicos científicos.

\section{Métodos}

Trata-se de uma revisão sistemática realizada conforme recomendações do Transparent Reporting of Systematic Reviews and Meta-Analyses (PRISMA). ${ }^{11}$ Para a identificação de estudos sobre o tema, foram pesquisadas as bases SciELO (Scientific Eletronic Library Online), LILACS (Literatura Latino-americana e do Caribe em Ciências da Saúde) e PubMed (U.S. National Library of Medicine) em maio de 2013, sem restrição de idioma, de período de publicação ou de população estudada (faixa etária ou outras características). Os seguintes critérios de inclusão foram adotados: ser estudo original com dados quantitativos e publicado em formato de artigo científico em periódico com corpo editorial e revisão por pares, ter sido realizado com população brasileira e apresentar dados sobre prevalência e/ou frequência de violência no ambiente escolar e/ou no trajeto de ida ou volta da escola. Definiram-se como critérios de exclusão: editoriais ou cartas ao editor, artigos de opinião, estudos de revisão, estudos com abordagem qualitativa, estudos sobre violência ocorrida exclusivamente fora do ambiente escolar e estudos não realizados no Brasil.

Os termos descritos a seguir foram utilizados para a localização das publicações, associados aos operadores lógicos “ $A N D$ ”, para relacionar termos, e "OR", para somar termos, e ao símbolo de truncagem * (asterisco), para pesquisar palavras de mesma raiz:

a) SciELO: a busca se deu por palavras do resumo combinando as palavras e operadores: (violência $O R$ agressão $O R$ agressividade $O R$ bullying $O R$ assédio) $A N D$ (professor $O R$ professores $O R$ aluno $O R$ alunos $O R$ escola $O R$ escolas $O R$ escolar $O R$ escolares $O R$ educação $O R$ ensino $O R$ colégio $O R$ estudante $O R$ estudantes) $A N D$ (estatística $O R$ estatísticas $O R$ estatístico $O R$ estatísticos $O R$ quantitativa $O R$ quantitativas $O R$ quantitativo $O R$ quantitativos $O R$ análise $O R$ análises $O R$ frequência $O R$ frequências $O R$ proporção $O R$ proporções $\mathrm{OR}$ percentual $O R$ percentuais $O R$ percentagem $O R$ porcentagem $O R$ amostra $O R$ distribuição $O R$ populacional $O R$ associação $O R$ medidas $O R$ associações $O R$ questionário $O R$ questionários $O R$ transversal $O R$ seccional $O R$ inquérito $O R$ coorte $O R$ risco $O R$ odds).

b) LILACS: os artigos também foram buscados por 
palavras do resumo, com as seguintes combinações: (violência $O R$ agressão $O R$ agressividade $O R$ bullying $O R$ assédio) $A N D$ (professor $O R$ professores $O R$ aluno $O R$ alunos $O R$ escola $O R$ escolas $O R$ escolar $O R$ escolares $O R$ educação $O R$ ensino $O R$ colégio $O R$ estudante $O R$ estudantes) $A N D$ (estatística $O R$ estatísticas $O R$ estatístico $O R$ estatísticos $O R$ quantitativa $O R$ quantitativas $O R$ quantitativo $O R$ quantitativos $O R$ análise $O R$ análises $O R$ frequência $O R$ frequências $O R$ proporção $O R$ proporções $O R$ percentual $O R$ percentuais $O R$ percentagem $O R$ porcentagem $O R$ amostra $O R$ distribuição $O R$ populacional $O R$ associação $O R$ medidas $O R$ associações $O R$ questionário $O R$ questionários $O R$ transversal $O R$ seccional $O R$ inquérito $O R$ coorte $O R$ risco $O R$ odds).

c) PubMed: a busca se deu por palavras contidas no resumo ou no título do artigo, com os seguintes operadores e truncagens: (violen* OR aggress* ${ }^{*}$ OR bullying OR harass* OR conflict* OR rage* OR embarrass* OR coercion* OR humiliation $O R$ provocation $O R$ insult* $O R$ vandalism*) $A N D$ (school* OR scholar OR educa* OR class* OR teach* OR student*). Além disso, adicionou-se "AND Brazil" a essa estratégia, com busca em todos os campos disponíveis nessa base.

A busca, análise e seleção dos artigos foram feitas de forma independente por duas pesquisadoras. A análise dos artigos foi realizada em três etapas: avaliação dos títulos, leitura dos resumos e leitura da versão completa. Após leitura completa dos artigos selecionados, foram verificadas as referências utilizadas nesses trabalhos a fim de identificar publicações não localizadas anteriormente e que fossem pertinentes ao tema da revisão.

Dado que o objetivo do estudo era realizar uma revisão ampliada sobre estudos quantitativos que abordassem a violência escolar no Brasil, e que o tema em questão poderia apresentar as mais diversas abordagens conceituais e metodológicas, não foram estabelecidos previamente critérios de qualidade para a seleção dos estudos. No entanto, optou-se por incluir apenas artigos submetidos ao escrutínio de editores e revisores de periódicos indexados em bases bibliográficas que estabelecem critérios para seleção e permanência de periódicos em sua base, tais como necessidade de Comitê Editorial, de revisão por pares dos artigos apresentados, periodicidade, entre outros.

Os artigos foram analisados em relação ao local e período de realização do estudo, delineamento, sujeitos pesquisados, dependência administrativa das escolas (pública ou privada), nível de ensino (fundamental ou médio) e natureza da violência. Esta última foi categorizada em física, sexual e psicológica, conforme classificação proposta pela Organização Mundial da Saúde. ${ }^{2}$ Também foram acrescentadas as categorias "contra o patrimônio/ material" e "geral". A primeira refere-se a atos como roubar, quebrar ou danificar materiais de instituições ou de pessoas, conforme definição de Stelko-Pereira e Williams. ${ }^{4}$ A segunda engloba casos em que a natureza não foi especificada ou quando houve agrupamento de violências de diferentes naturezas. O bullying, por envolver atos violentos de diferentes naturezas, e por ser uma das violências mais estudadas no contexto da escola, foi considerado como modalidade específica da violência escolar.

A violência observada pelo entrevistado no ambiente escolar foi considerada como frequência, ou seja, mais de um entrevistado poderia referir a mesma situação de violência. A prevalência foi caracterizada como exposição ao ato violento, seja na condição de vítima, autor, ou vítima/autor em relação ao total dos investigados de cada estudo. Medidas de frequência e de prevalência, quando não explicitamente apresentadas nos artigos, foram calculadas pelos autores desta revisão, tendo como base os dados disponíveis em texto ou tabelas dos estudos analisados. Nos artigos de associação, foram analisadas as medidas de associação apresentadas (odds ratio, razão de prevalência ou risco relativo, e respectivos intervalos de confiança de $95 \%$ ou valor de p para avaliar significância estatística).

Nos estudos que buscaram associação entre violência e algumas características optou-se por apresentar resultados de variáveis cuja associação apresentou valor de $p<0,05$ na análise bivariada ou na multivariada, quando disponível.

\section{Resultados}

Foram identificados 1174 artigos, de acordo com estratégia de busca adotada. Desses, 1134 foram excluídos por informações contidas no título, após leitura do resumo e por duplicação nas bases de dados. Após leitura completa dos 40 artigos restantes, excluíram-se 17. Somou-se um estudo mencionado em referência de um dos artigos selecionados para leitura completa e que atendia aos critérios de inclusão, resultando em amostra final de 24 artigos científicos (Figura 1).

Aproximadamente $80 \%$ dos estudos foram publicados nos últimos cinco anos (2009-2013)12-30 e no idioma português.12-27,31-33 Os estudos tiveram como principal localização geográfica as Regiões Sudeste e Sul do Brasil, com destaque para os Estados de São Paulo12,14,16,19,21,23 e Rio Grande do 


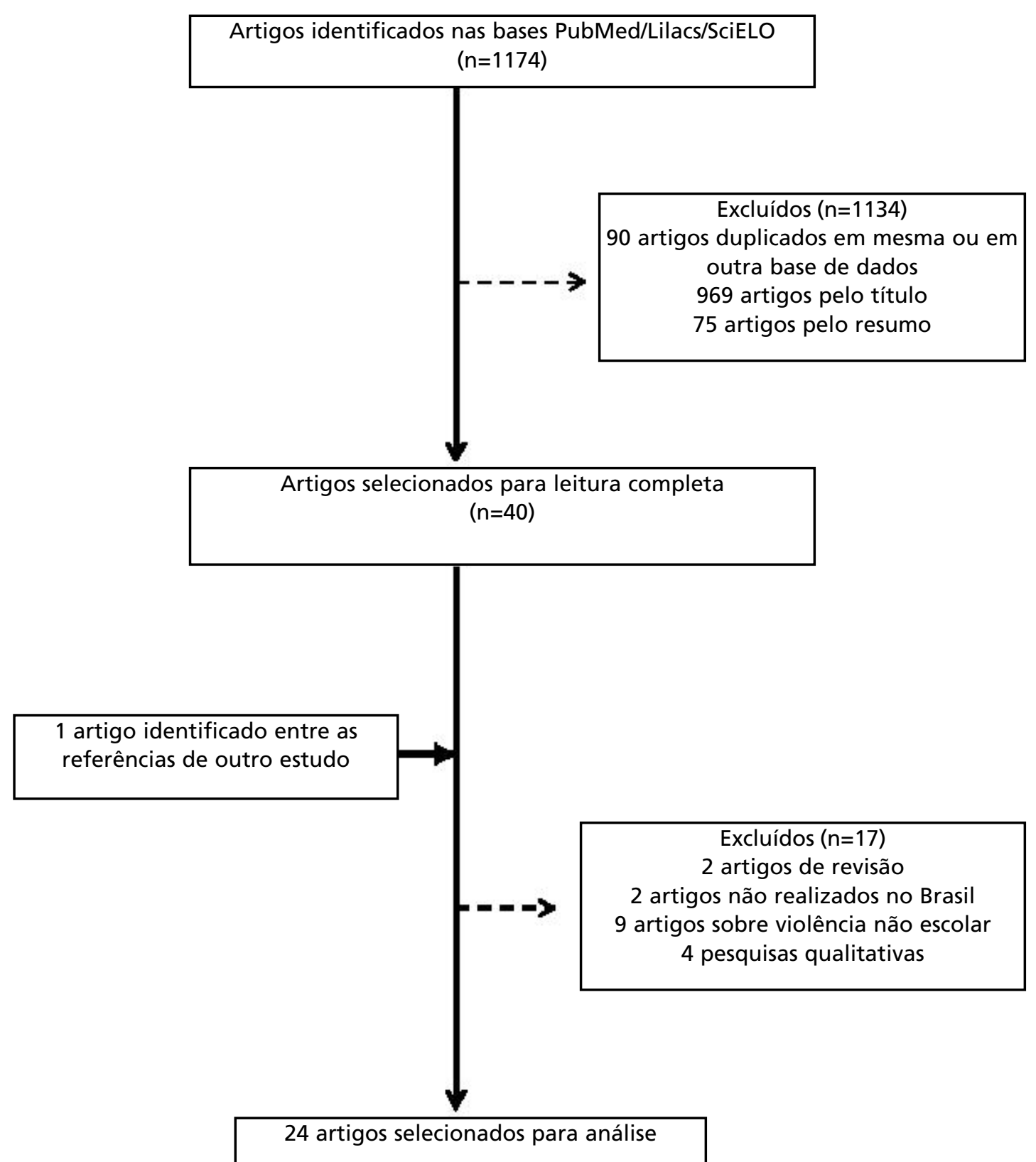

Sul, 13,22,24,26,29,30 com seis estudos cada. Um artigo ${ }^{9}$ analisou cinco capitais e o Distrito Federal, englobando todas as regiões brasileiras, e outros três $17,18,25$ investigaram as 26 capitais e o Distrito Federal.

A maioria das pesquisas foi realizada em escolas públicas, com alunos do ensino fundamental. Em 19 estudos a população foi composta por alunos, 9,12 15,17-19,21,22,24-27,29-32,34 e em cinco $16,20,23,28,33$ por professores (Tabela 1). O tamanho amostral variou de 30 (professores) até 60.973 (alunos do ensino fundamental e médio de escolas públicas e particulares). Não foi localizado nenhum estudo que pesquisou, simultaneamente, alunos e professores. Todas as pesquisas apresentaram delineamento transversal, com obtenção de dados por meio de questionários.

A Tabela 2 apresenta resultados sobre 
prevalência de violência escolar. Entre os estudos realizados com alunos, cuja natureza da violência foi especificada, o bullying12-14,17,22,24-27,29,30,34 foi o mais pesquisado, seguido da violência psicológica14,18,19,32 e da física.9,18,21,32 A prevalência de vítimas de bullying variou de $10,2 \% 29$ a $31,1 \%, 25$ a de autores de bullying de 2,9\%12 a $60,0 \% 34$ e a de vítimas/autores esteve entre $8,3 \% 27 \mathrm{e}$ $70,0 \% .13$ As prevalências de violência psicológica e de violência física estiveram, respectivamente, entre $5,5 \%$ (sensação de insegurança na escola, resultando em faltas às aulas) 18 e 70,0\% (autor de xingamentos) ${ }^{19}$ e entre 3,6\% (vítima de agressão física) ${ }^{32}$ e $12,9 \%$ (participação em brigas envolvendo agressão física). ${ }^{18}$

Apenas dois artigos realizados com alunos 9,32 pesquisaram a violência contra o patrimônio/material, com prevalências de $2,8 \%$ (vítima de furto de dinheiro) 32 e de $38,2 \%$ (vítima de furto), 9 e apenas um, 34 a violência sexual, em que $24,0 \%$ dos alunos afirmaram ser perpetradores de assédio sexual (Tabela 2).

Três estudos realizados com professores apresentaram dados de prevalência. Em apenas um a natureza da violência foi especificada e verificaramse as seguintes prevalências: insultos verbais $(76,5 \%)^{20}$ (violência psicológica), extorsão $(6,9 \%), 20$ furto ou danificação de pertences $(27,5 \%)^{20}$ (violência contra o patrimônio/material) e assédio sexual $(30,4 \%)^{20}$ (violência sexual) (Tabela 2).

As pesquisas com alunos apontaram locais, no ambiente escolar, ou contextos em que há maior ocorrência de violência: pátio da escola22,29 e sala de aula, 13,14,24,29 durante o recreio, 14,24 entrada ou saída da escola, 24 e no caminho de ida ou volta da escola. ${ }^{29}$ Entre os professores, ${ }^{20}$ houve relato de maior insegurança na quadra de esportes e no portão de entrada das escolas.

Houve delimitação do período de exposição à violência em 12 estudos. A violência geral foi delimitada em 30 dias, 18 seis meses 21 e um ano, 28 e o bullying em 30 dias 17,22,25,34 ou em outros intervalos de tempo (última semana, últimos três meses, último ano, mais de um ano). 12,14,24,27,29

As categorias de respostas referentes à frequência das ações violentas e sua classificação como bullying variaram em número e especificidade. Alguns estudos consideraram, para o estabelecimento da frequência, categorias mais abrangentes como as que envolveram "nenhuma vez/nunca" até "sempre/frequentemente".17,25,30 Outros optaram por fazer referência temporal ao número de atos violentos (ocorrências por semana, mês, ano).12,14,24
Oito estudos $9,13,16,19,20,23,24,26$ apresentaram dados de frequência. A existência de violência na escola foi relatada por $83,4 \%$ dos alunos 9 e por $87,3 \%$ dos professores. ${ }^{20} \mathrm{O}$ preconceito foi referido por $76,0 \%$ dos alunos 13 e $93,3 \%$ dos professores afirmaram já ter presenciado algum episódio de discriminação na escola.16 Ser testemunha de bullying oscilou entre $82,0 \% 24$ e $83,9 \% 26$ (Tabela 3 ).

Dos 24 artigos analisados, 1512,20-26,28-34 apresentaram resultados de associação entre violência escolar e algumas características. O bullying representou a violência mais abordada nos estudos de associação realizados com alunos, 12,22,24,26,27,29,30,34 e ser do sexo masculino12,26,29,30,34 foi a característica mais frequentemente associada à condição de autor ou de vítima/autor de bullying (Tabela 4).

Apenas dois estudos identificaram associação entre ter vivenciado situações de violências na família12,31 e ser vítima de violências no ambiente escolar (Tabela 4).

Três artigos objetivaram verificar a existência de associação entre vivência de violência nas escolas e problemas relacionados à saúde do professor. A prevalência de transtornos mentais associou-se a ter sido agredido por alunos, pais de alunos ou pessoas externas à escola. ${ }^{33}$ Associaram-se à referência de distúrbios de voz: ameaças ao professor, agressões, insultos e manifestações de racismo na escola, violência à porta da escola e violência contra funcionários. ${ }^{23} \mathrm{~A}$ exposição à violência praticada por alunos ou pais de alunos esteve associada ao absenteísmo por distúrbio de voz28 (Tabela 4).

\section{Discussão}

O presente estudo detectou poucos artigos científicos quantitativos sobre violência escolar no Brasil, bem como aplicação de instrumentos variados para sua detecção. Mais recentemente, como mostra esta revisão, houve incremento da contribuição científica sobre o tema no país, possivelmente em decorrência da atenção direcionada às novas formas de violência na escola: depredações, invasões de espaços escolares e brigas entre grupos foram acrescidas de agressões de alunos contra professores, uso de arma branca e de fogo, consumo de drogas, preconceito e bullying. 35

A falta de consenso na definição de violência e na padronização para a elaboração dos instrumentos nos estudos analisados dificultou a sistematização dos resultados desta revisão. Dados quantitativos sobre violência escolar são influenciados pela definição, período de tempo e local considerado para seu cálculo.36 A sistematização do conceito de 


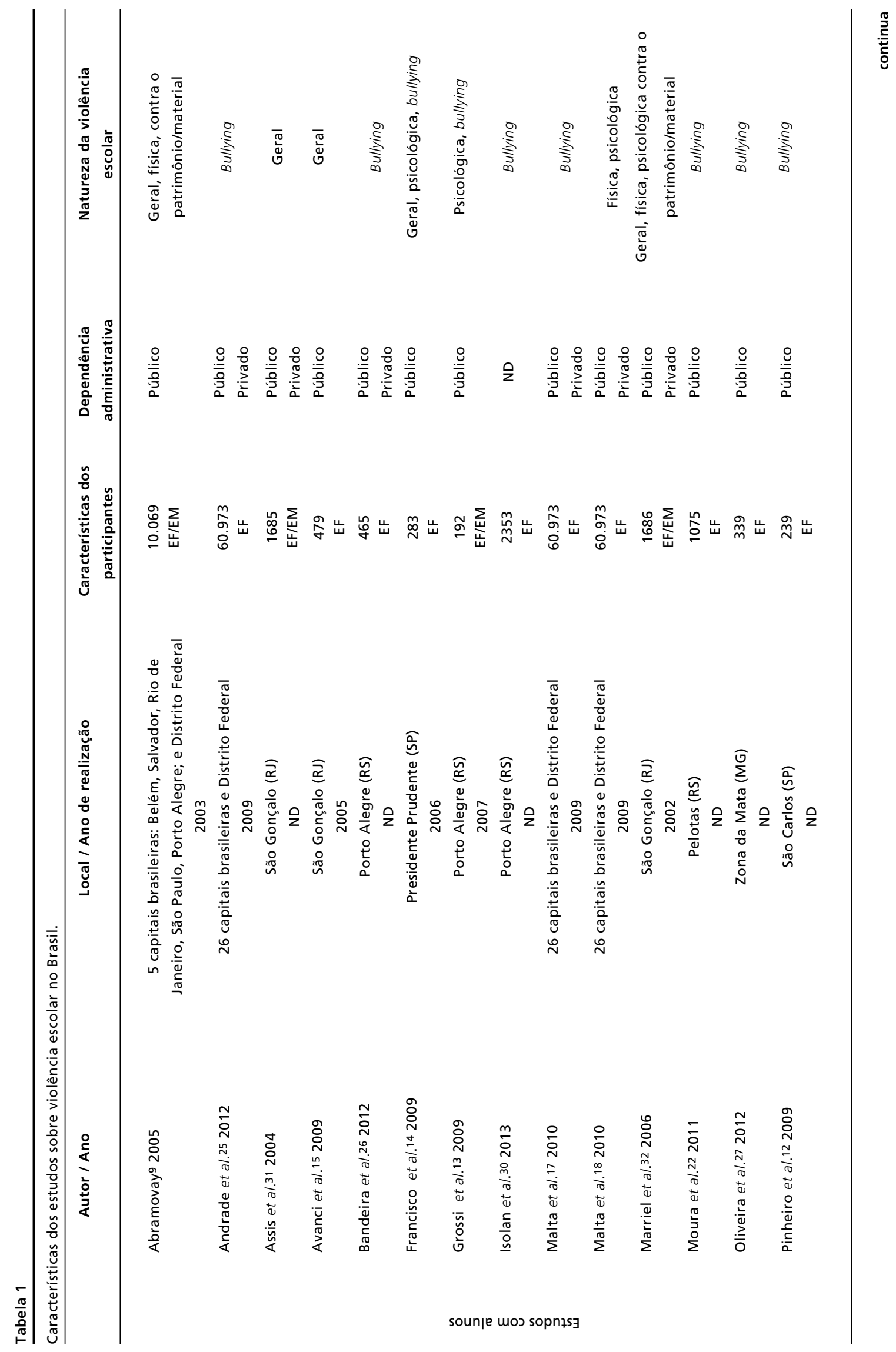




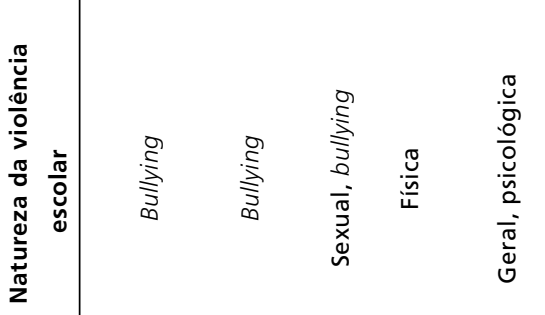

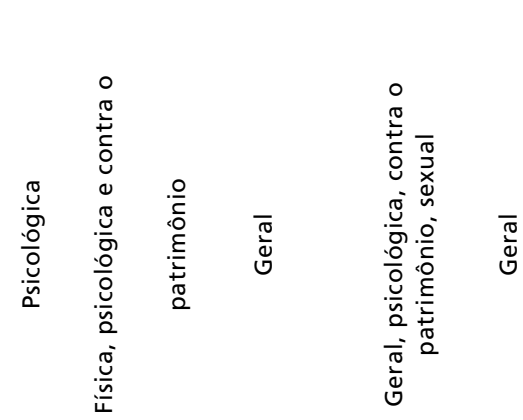

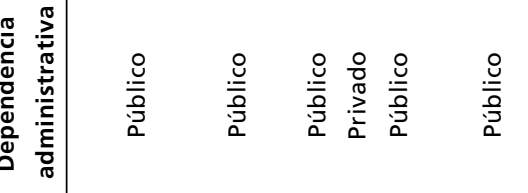

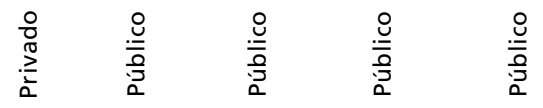

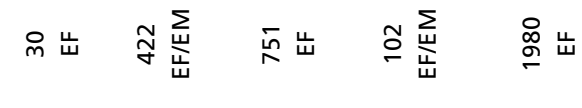

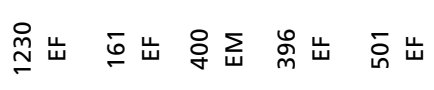

$\frac{10}{30}$



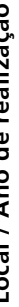







$\checkmark$

$\sim$

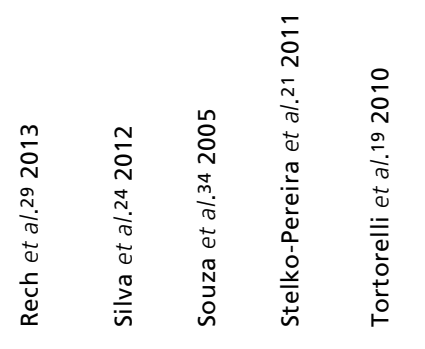

$$
\begin{aligned}
& \text { (oé̃ } \\
& \text { sounje mos sopnłs }
\end{aligned}
$$

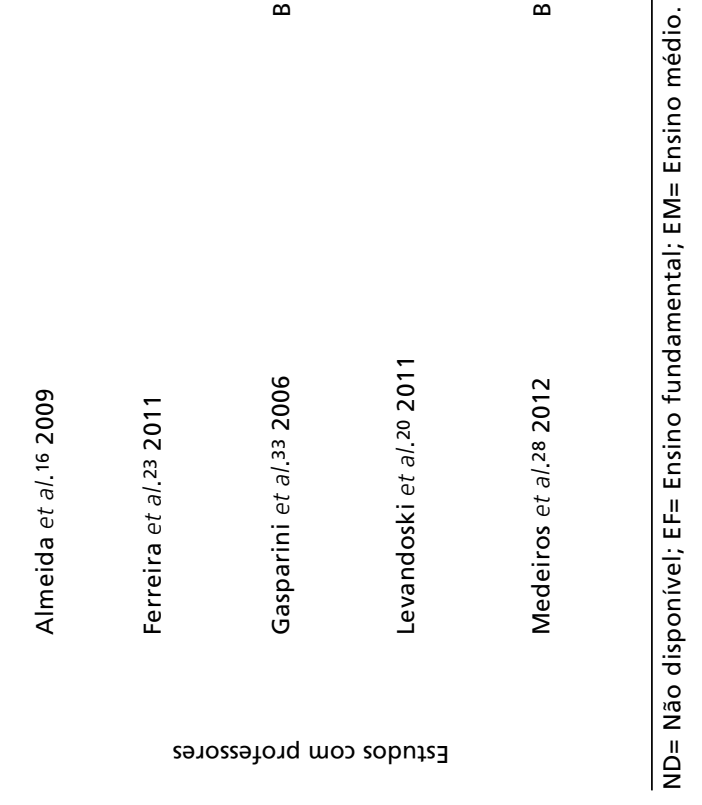














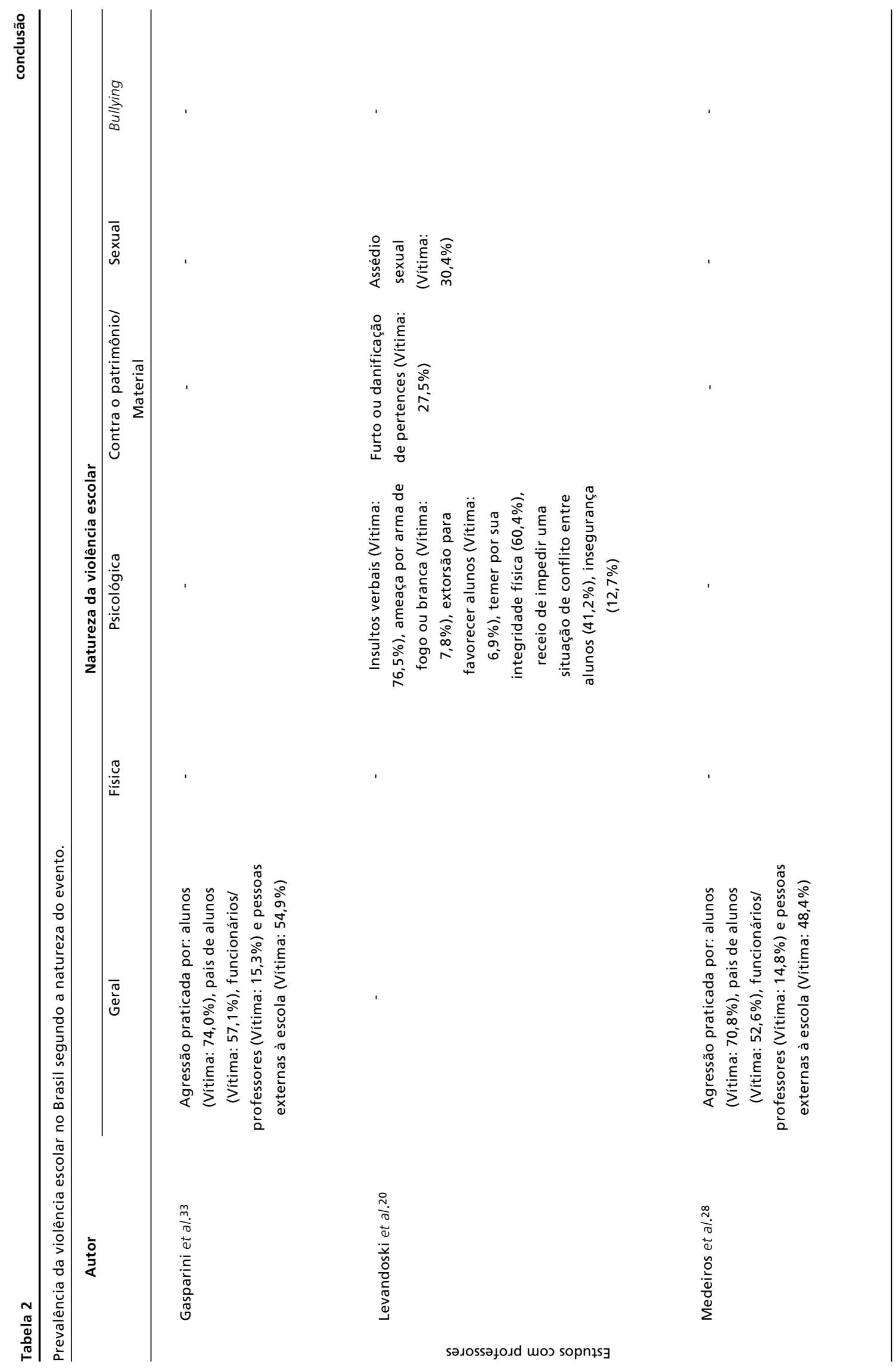




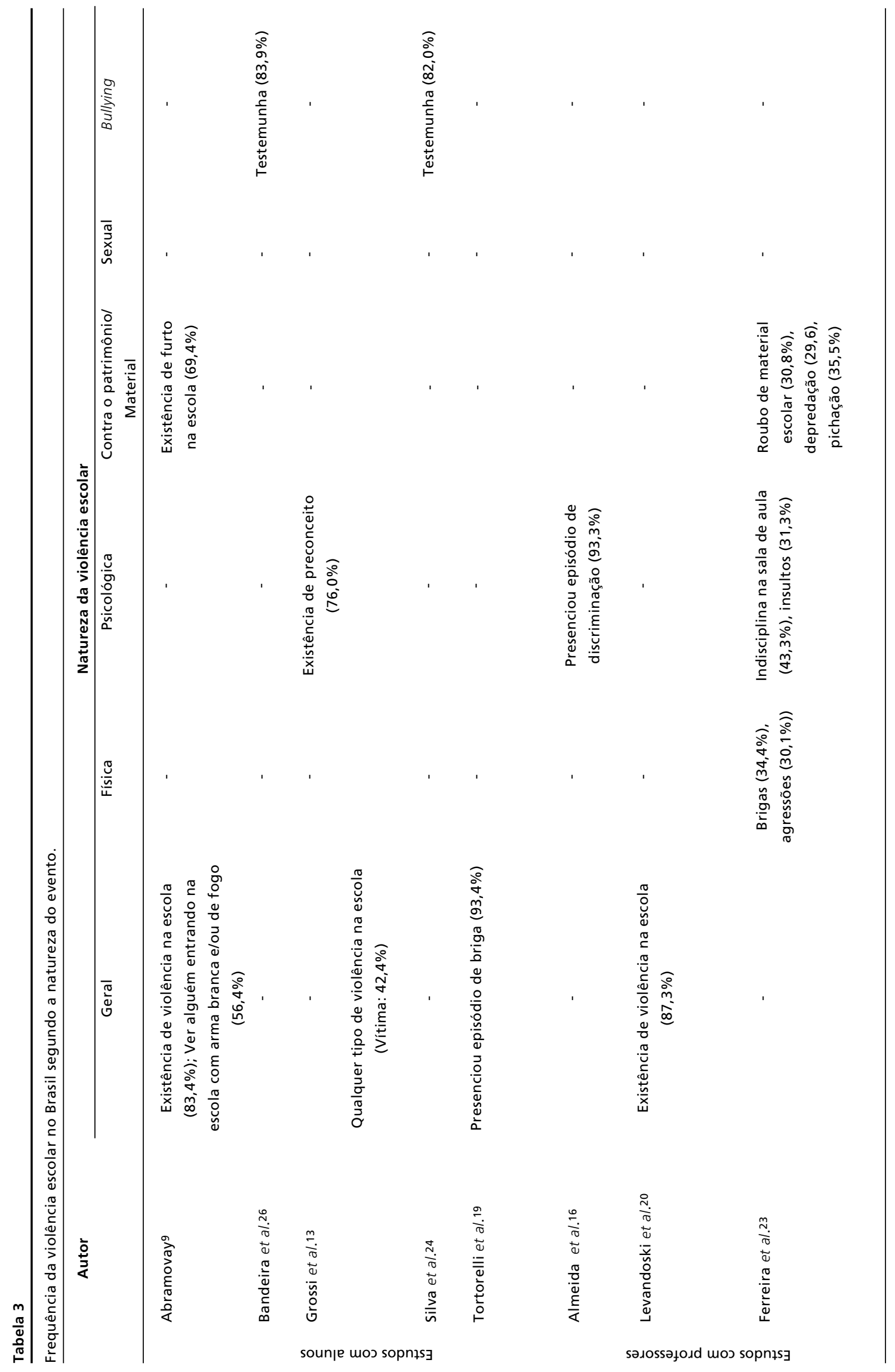




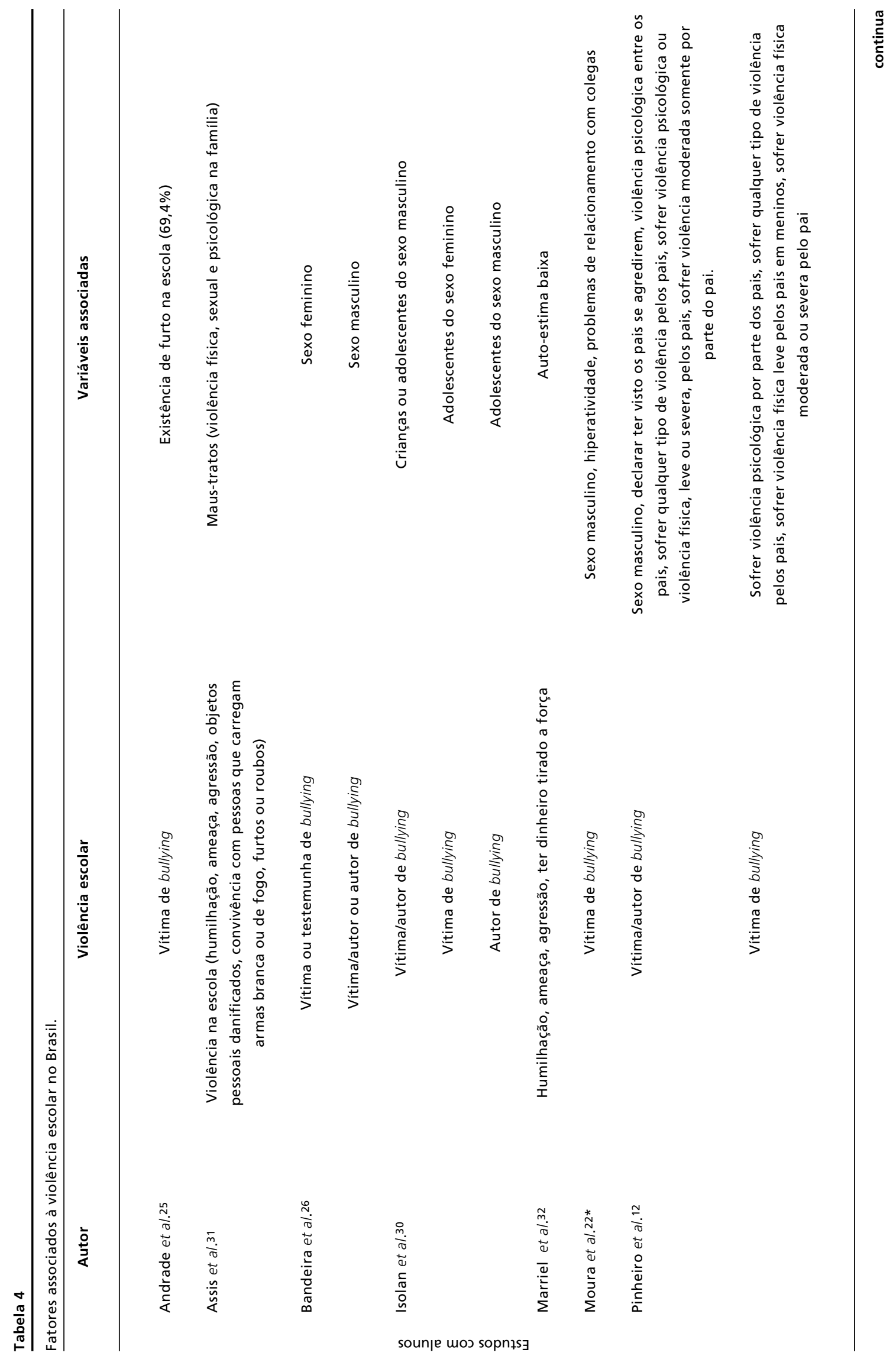




Nesello F et al.



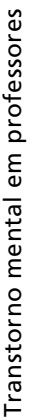

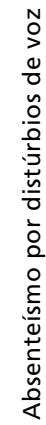

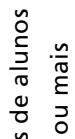

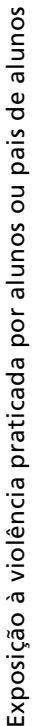

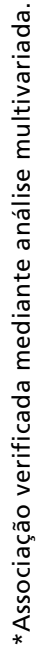


violência escolar é essencial para o estabelecimento de diálogo entre pesquisadores e para a construção de instrumento de referência para coleta de dados que possibilite maior nível de detalhamento e auxilie na compreensão desse fenômeno.

Além do viés da ausência de consenso sobre o conceito de violência escolar, outro aspecto que merece destaque é o delineamento transversal de todos os estudos selecionados, possibilitando a existência do viés de sobrevivência, isto é, vítimas de violência escolar que abandonaram a escola ou que não estavam presentes nos dias de coleta de dados, não foram captadas pelos estudos.

Acrescente-se que como não foram analisados potenciais vieses de cada trabalho selecionado, presume-se que a validade externa dos resultados seja limitada, pois grande parte pesquisou estudantes ou professores de escolas específicas, reduzindo a capacidade de extrapolação dos achados. No entanto, esta revisão traz uma síntese importante dos estudos publicados sobre a temática no Brasil, possibilitando destacar e comentar alguns desses.

Observou-se que metade dos estudos foi realizada nos Estados de São Paulo e Rio Grande do Sul e a maioria das pesquisas investigou alunos do ensino fundamental, evidenciando que os do ensino médio ainda são pouco pesquisados no Brasil. Esse resultado pode estar associado ao consenso de que o relato de violência é mais frequente entre os mais jovens. 37-39 Pesquisas com alunos do ensino médio de outros países evidenciam que, além da redução na frequência da violência com o aumento do nível de ensino, 40,41 podem ocorrer mudanças em sua percepção, decorrentes do desenvolvimento psicológico e cognitivo, bem como de novas experiências sociais e demandas acadêmicas. ${ }^{37}$ Felix $e t$ $a l .42$ verificaram maior frequência de vitimização física entre alunos do ensino fundamental, e predomínio de assédio sexual entre alunos do ensino médio.

Apesar de pouco percebida, ${ }^{43}$ a violência psicológica tem prevalências e frequências altíssimas nas escolas. Ter sido ameaçado, ${ }^{14,19,32}$ humilhado, ${ }^{32}$ desprezado 19 ou vítima de bullying12,14,17,22,24-27,29,30 foram aspectos verificados por mais da metade dos estudos realizados com alunos, nesta revisão. Além disso, existência de preconceito $^{13}$ e de discriminação 16 na escola apresentou frequências elevadas (respectivamente 76,0\% e 93,3\%). Faltas à escola nos 30 dias anteriores à pesquisa por sensação de insegurança nesse ambiente foram relatadas por $5,5 \%$ dos estudantes de capitais dos Estados brasileiros e do Distrito Federal,18 o que revela a percepção de vulnerabili- dade desses adolescentes à violência escolar e a consequência nefasta de afastá-los da escola por essa sensação. Essas vivências podem trazer implicações negativas às relações sociais estabelecidas na escola e à saúde mental dos indivíduos, como isolamento social e sintomas de estresse, ${ }^{44}$ ansiedade e depressão. $45-47$

Verificaram-se desigualdades na exposição a fatores de risco para violência escolar segundo o contexto social. Malta et al.17 destacam que a sensação de insegurança e brigas com arma branca e de fogo ocorrem mais frequentemente entre alunos de escolas públicas, em geral provenientes de famílias de menor poder aquisitivo em comparação aos alunos das particulares. De modo semelhante, D’Angelo e Fernández ${ }^{48}$ verificam, em estudo realizado em Buenos Aires e região metropolitana, na Argentina, diferenças nos tipos de violência de acordo com a classe social. Enquanto o bullying foi mais frequente em escolas privadas, o porte de arma de fogo foi mais observado em escolas públicas, frequentadas por estudantes de classes sociais elevadas e baixas, respectivamente.

A presente revisão detectou poucos estudos de fatores associados à violência escolar. Foram investigados, principalmente, fatores associados ao bullying e à saúde do professor. Ser do sexo masculino foi o fator de maior destaque para vitimização e/ou perpetração de bullying. Esse resultado também foi observado em estudos realizados em outros países49-51 e assinala para o papel de práticas machistas e de risco na configuração do gênero masculino, e de sua condição de vítima e autor da violência. 52,53

Fatores individuais, de relacionamentos estabelecidos, comunitários e sociais atuam conjuntamente para a expressão da violência em diferentes espaços sociais. ${ }^{1}$ Ter vivenciado violência no âmbito familiar associou-se à violência escolar em dois estudos desta revisão,12,31 assinalando para a estreita relação existente entre violências de várias fontes e naturezas. Njaine e Minayo 54 afirmam que a negação do diálogo e as formas de violência física, sexual e psicológica contra crianças e adolescentes que ocorrem no âmbito intrafamiliar podem refletir na vida escolar sob a forma de comportamentos agressivos ou apáticos dos alunos.

Os estudos sobre fatores associados à saúde do professor evidenciaram relação da violência com consequências físicas, emocionais e psicológicas. Além disso, outras pesquisas destacam impactos negativos da violência contra o professor no ensino e no engajamento profissional, causando prejuízos não somente à sua saúde, mas também ao processo de 
educação de crianças e adolescentes. 6,55 A preocupação com a saúde do professor mostra que o seu adoecimento pode estar relacionado ao contexto profissional, que engloba fatores como condições negativas e problemas de organização do trabalho, relações sociais estressantes e exposição a ambientes conflituosos, permeados por agressividade, indisciplina, desrespeito e violência. 56,57

A abordagem qualitativa da violência escolar tem trazido importantes contribuições para o entendimento desse fenômeno.43,54,58 Todavia, estudos quantitativos também são necessários, na medida em que produzem dados sobre morbidade, mortalidade, consequências da violência, fatores que aumentam o risco de ser vítima ou perpetrador de crimes e agressões, bem como configurações locais e especificações demográficas dos eventos violentos. 59

Em síntese, observou-se distribuição desigual dos estudos entre diferentes regiões do país e, embora ainda em pequeno número, aumento de publicações em anos recentes. A heterogeneidade de métodos, de critérios para definição de violência

\section{Referências}

1. Assis SG, Marriel LC. Reflexões sobre violência e suas manifestações na escola - um diálogo com professores. In: Assis SG, Constantino P, Avanci JQ. Impactos da violência na escola. Rio de Janeiro: Fiocruz; 2010. p. 41-63.

2. Krug EG, Dahlberg LL, Mercy JA, Zwi AB, Lozano R. World report on violence and health Geneva: Word Health Organization; 2002.

3. Martins CBG, Mello Jorge MHP. Violência contra crianças e adolescentes: contexto e reflexões sob a ótica da saúde. Londrina: EDUEL; 2011.

4. Stelko-Pereira AC, Williams LCA. Reflexões sobre o conceito de violência escolar e a busca por uma definição abrangente. Temas psicol. 2010; 18: 45-55.

5. Abramovay M. Violencia en las escuelas: un gran desafio. Rev Iberoamericana Educ. 2005; 38: 53-66.

6. Wilson CM, Douglas KS, Lyon DR. Violence against teachers: prevalence and consequences. $\mathrm{J}$ Interpers Violence. 2011; 26: 2353-71.

7. Akiba M, LeTendre GK, Baker DP, Goesling B. Student victimization: national and school system effects on school violence in 37 nations. Am Educ Res J. 2002; 39: 829-53.

8. Chen JK, Astor RA. School violence in Taiwan: examining how Western risk factors predict school violence in an Asian culture. J Interpers Violence. 2010; 25: 1388-410.

9. Abramovay M. Victimización en las escuelas: ambiente escolar, robos y agresiones físicas. Rev Mex Invest Educ. 2005; 10: 833-64.

10. Galvão A, Gomes CA, Capanema C, Caliman G, Câmara J. Violências escolares: implicações para a gestão e o currículo. Ensaio: Aval Pol Públ Educ. 2010; 18: 425-42. escolar e de populações estudadas, no entanto, dificultaram a sistematização dos resultados dos artigos analisados nesta revisão. Pode-se destacar, porém, que esse fenômeno apresentou prevalência e frequência elevadas e esteve associado a fatores que trazem implicações à integridade física e psíquica de professores e de alunos. Associou-se, ainda, a características sociodemográficas e a violências sofridas em outros espaços sociais. Há necessidade de ampliar o diálogo nacional entre pesquisadores do tema para estabelecimento de medidas padronizadas para o estudo desse fenômeno, de forma a permitir comparações no tempo e entre diferentes localidades, além de possibilitar a identificação de situações que aumentam a vulnerabilidade à violência no ambiente escolar.

\section{Agradecimentos}

À Fundação Araucária, pelo auxílio financeiro em forma de Bolsa Técnico. À CAPES e ao CNPq, pela concessão de bolsas.

11. Moher D, Liberati A, Tetzlaff J, Altman DG. Preferred reporting items for systematic reviews and meta-analyses: the PRISMA statement. BMJ. 2009; 339: b2535.

12. Pinheiro FMF, Williams LCA. Violência intrafamiliar e intimidação entre colegas no ensino fundamental. Cad Pesqui. 2009; 39: 995-1018.

13. Grossi PK, Santos AM. Desvendando o fenômeno bullying nas escolas públicas de Porto Alegre, RS, Brazil. Rev Portug Educ. 2009; 22: 249-67.

14. Francisco MV, Libório RMC. Um estudo sobre bullying entre escolares do ensino fundamental. Psicol Reflex Crit. 2009; 22: 200-7.

15. Avanci J, Assis S, Oliveira R, Pires T. Quando a convivência com a violência aproxima a criança do comportamento depressivo. Cien Saúde Colet. 2009; 14: 383-94.

16. Almeida SB, Cardoso LRD, Costac VV. Bullying: conhecimento e prática pedagógica no ambiente escolar. Psicol Argum. 2009; 27: 201-6.

17. Malta DC, Silva MAI, Mello FCM, Monteiro RA, Sardinha LMV, Crespo C, Carvalho MGO, Silva MMA, Porto DL. Bullying nas escolas brasileiras: resultados da Pesquisa Nacional de Saúde do Escolar (PeNSE), 2009. Ciênc Saúde Coletiva. 2010; 15: 3065-76.

18. Malta DC, Souza ER, Silva MMA, Silva CS, Andreazzi MAR, Crespo C, Mascarenhas MDM, Porto DL, Figueroa ALG, Morais Neto OL, Penna GO. Vivência de violência entre escolares brasileiros: resultados da Pesquisa Nacional de Saúde do Escolar (PeNSE). Ciênc Saúde Coletiva. 2010; 15: 3053-63. 
19. Tortorelli MFP, Carreiro LRR, Araújo MV. Correlações entre a percepção da violência familiar e o relato de violência na escola entre alunos da cidade de São Paulo. Psicol Teor Prát. 2010; 12: 32-42.

20. Levandoski G, Ogg F, Cardoso FL. Violência contra professores de educação física no ensino público do estado do Paraná. Motriz Rev Educ Fís. 2011; 17: 374-83.

21. Stelko-Pereira AC, Santini PM, Williams LCA. Punição corporal aplicada por funcionários de duas escolas públicas brasileiras: prevalence in two public schools. Psicol Estud. 2011; 16: 581-91.

22. Moura DR, Cruz ACN, Quevedo LA. Prevalência e características de escolares vítimas de bullying. J Pediatr (Rio J). 2011; 87: 19-23.

23. Ferreira LP, Latorre MRDO, Giannini SPP. A violência na escola e os distúrbios de voz de professores. Disturb Comun. 2011; 23: 165-72.

24. Silva CE, Oliveira RV, Bandeira DR, Souza DO. Violência entre pares: um estudo de caso numa escola pública de Esteio/RS. Psicol Esc Educ. 2012; 16: 83-93.

25. Andrade SSCA, Yokota RTC, Sá NNB, Silva MMA, Araújo WN, Mascarenhas MDM, Malta DC. Relação entre violência física, consumo de álcool e outras drogas e bullying entre adolescentes escolares brasileiros. Cad Saúde Pública. 2012; 28: 1725-36.

26. Bandeira CM, Hutz CS. Bullying: prevalência, implicações e diferenças entre os gêneros. Psicol Esc Educ. 2012; 16: 35-44.

27. Oliveira JC, Barbosa AJG. Bullying entre estudantes com e sem características de dotação e talento. Psicol Reflex Crít. 2012; 25 : 747-55.

28. Medeiros AM, Assuncao AA, Barreto SM. Absenteeism due to voice disorders in female teachers: a public health problem. Int Arch Occup Environ Health. 2012; 85: 85364.

29. Rech RR, Halpern R, Tedesco A, Santos DF. Prevalence and characteristics of victims and perpetrators of bullying. $\mathrm{J}$ Pediatr (Rio J). 2013; 89: 164-70.

30. Isolan L, Salum GA, Osowski AT, Zottis GH, Manfro GG. Victims and bully-victims but not bullies are groups associated with anxiety symptomatology among Brazilian children and adolescents. Eur Child Adolesc Psychiatry. 2013; 22 (10): 641-8.

31. Assis SG, Avanci JQ, Santos NC, Malaquias JV, Oliveira RVC. Violência e representação social na adolescência no Brasil. Rev Panam Salud Publica. 2004; 16: 43-51.

32. Marriel LC, Assis SG, Avanci JQ, Oliveira RVC. Violência escolar e auto-estima de adolescentes. Cad Pesqui. 2006; 36: $35-50$

33. Gasparini SM, Barreto SM, Assunção AA. Prevalência de transtornos mentais comuns em professores da rede municipal de Belo Horizonte, Minas Gerais, Brasil. Cad Saúde Pública. 2006; 22: 2679-91.

34. Souza ER, Ribeiro J. Bullying and sexual harassment among Brazilian high school students. J Interpers Violence. 2005; 20: 1018-38.

35. Castro ML, Cunha SS, Souza DPO. Comportamento de violência e fatores associados entre estudantes de Barra do Garças, MT. Rev Saúde Pública. 2011; 45: 1054-61.
36. Solberg ME, Olweus D. Prevalence estimation of school bullying with the olweus bully/victim questionnaire. Aggressive Behav. 2003; 29: 239-68.

37. Craig W, Harel-Fisch Y, Fogel-Grinvald H, Dostaler S, Hetland J, Simons-Morton B, Molcho M, Mato MG, Overpeck M, Due P, Pickett W, HBSC Violence \& Injuries Prevention Focus Group, HBSC Bullying Writing Group. A cross-national profile of bullying and victimization among adolescents in 40 countries. Int J Public Health. 2009; 54: 216-24.

38. Lopes Neto AA. Bullying: comportamento agressivo entre estudantes. J Pediatr (Rio J). 2005; 81: 164-72.

39. Ristum M. Bullying Escolar. In: Assis SG, Constantino P, Avanci JQ. Impactos da violência na escola - um diálogo com professores. Rio de Janeiro: Fiocruz; 2010. p. 95-120.

40. Chen JK, Astor RA. The perpetration of school violence in Taiwan: an analysis of gender, grade level and school type. School Psychol Int. 2009; 30: 568-84.

41. Scheithauer H, Hayer T, Petermann F, Jugert G. Physical, verbal, and relational forms of bullying among German students: age trends, gender differences, and correlates. Aggressive Behav. 2006; 32: 261-75.

42. Felix ED, Furlong MJ, Austin G. A cluster analytic investigation of school violence victimization among diverse students. J Interpers Violence. 2009; 24: 1673-95.

43. Abramovay M, Cunha AL, Calaf PP. Revelando tramas, descobrindo segredos: violência e convivência nas escolas. Brasília: Rede de Informação Tecnológica Latino-americana, Secretaria de Estado de Educação do Distrito Federal; 2009. 496 p.

44. Newman ML, Holden GW, Delville Y. Isolation and the stress of being bullied. J Adolesc. 2005; 28: 343-57.

45. Craig WM. The relationship among bullying, victimization, depression, anxiety, and aggression in elementary school children. Pers Individ Dif. 1998; 24: 123-30.

46. Chen X, Huang X, Wang L, Chang L. Aggression, peer relationships, and depression in Chinese children: a multiwave longitudinal study. J Child Psychol Psychiatry. 2012; 53: 1233-41.

47. Seals D, Young J. Bullying and victimization: prevalence and relationship to gender, grade level, ethnicity, selfesteem, and depression. Adolescence. 2003; 38: 735-47.

48. D'Angelo LA, Fernández DR. Clima, conflictos y violencia en la escuela Buenos Aires: Fondo de las Naciones Unidas para la Infancia - Facultad Latinoamericana de Ciencias Sociales; 2011.

49. Morbidity and Mortality Weekly Report. Bullying among middle school and high school students. Massachusetts: Centers for Disease Control and Prevention; 2009.

50. Khoury-Kassabri M, Astor RA, Benbenishty R. Middle Eastern adolescents' perpetration of school violence against peers and teachers: a cross-cultural and ecological analysis. J Interpers Violence. 2009; 24: 159-82.

51. Strawhacker MT. School violence: an overview. J Sch Nurs. 2002; 18: 68-72.

52. Landstedt E, Gillander GK. Experiences of violence among adolescents: gender patterns in types, perpetrators and associated psychological distress. Int J Public Health. 2011; 56: 419-27. 
53. Souza ER. Masculinidade e violência no Brasil: contribuições para a reflexão no campo da saúde. Ciênc Saúde Coletiva. 2005; 10: 59-70.

54. Njaine K, Minayo MCS. Violência na escola: identificando pistas para a prevenção. Interface: Comunic, Saúde, Educ. 2003; 7: 119-34.

55. Galand B, Lecocq C, Philippot P. School violence and teacher professional disengagement. Br J Educ Psychol. 2007; 77: 465-77.

56. Penteado RZ, Pereira IMTB. Qualidade de vida e saúde vocal de professores. Rev Saúde Pública. 2007; 41: 236-43.
57. Brum LM, Azambuja CR, Rezer JFP, Temp DS, Carpilovsky CK, Lopes LF, Schetinger MRC. Qualidade de vida dos professores da área de ciências em escola pública no Rio Grande do Sul. Trab, Educ Saúde. 2012; 10: 125-45.

58. Cruz GV, Pereira WR. Diferentes configurações da violência nas relações pedagógicas entre docentes e discentes do ensino superior. Rev Bras Enferm. 2013; 66: 241-50.

59. Minayo MCS. Violência e saúde. Rio de Janeiro: Fiocruz; 2006.

Recebido em 1 de novembro de 2013

Versão final apresentada em 27 de março de 2014

Aprovado em 2 de abril de 2014 